\title{
Severe Convective Weather Nowcasting System in Heilongjiang Meteorological Bureau and Its Preliminary Performance Evaluation
}

\author{
Xingmin Guan ${ }^{1}$, Xiang Zong² \\ ${ }^{1}$ Meteorological Data Center of Heilongjiang Meteorological Bureau, Heilongjiang, China \\ ${ }^{2}$ Information Technologies Co., (Beijing) Ltd., Beijing, China \\ Email: zongxiang@itcchina.com
}

How to cite this paper: Guan, X.M. and Zong, X. (2019) Severe Convective Weather Nowcasting System in Heilongjiang Meteorological Bureau and Its Preliminary Performance Evaluation. Atmospheric and Climate Sciences, 9, 323-330.

https://doi.org/10.4236/acs.2019.93023

Received: April 16, 2019

Accepted: July 2, 2019

Published: July 5, 2019

Copyright $\odot 2019$ by author(s) and Scientific Research Publishing Inc. This work is licensed under the Creative Commons Attribution International License (CC BY 4.0).

http://creativecommons.org/licenses/by/4.0/

\begin{abstract}
In this paper, we present the severe convective weather nowcasting system in Heilongjiang Meteorological Bureau and its preliminary performance evaluation, which has been in operational use since 2018. We believe that a good workflow design and intelligently integrated system plays an important role in the weather forecast service. Based on the current data environment, the system builds a unified data environment. Both the functions of automatic nowcasting system and Very-short-range Interactive Prediction System are presented too. The results show that this system plays a key role in the production of rapidly updated, convective storm nowcasting for end users.
\end{abstract}

Keywords

RMAPS-IN, VIPS, CIMISS

\section{Introduction}

There is a critical need for rapidly updating, accurate, high-resolution, short-term 0 - 6-h forecasts (nowcasts1) of convective precipitation that can be used in alerting the public of high-impact weather. The operational nowcast and forecast system in Heilongjiang Meteorological Bureau consists of several platforms and systems, including Rapid-refresh Multi-scale Analysis and Prediction System-INtegration (RMAPS-IN), Very-short-range Interactive Prediction System (VIPS) [1] and Automatic Nowcasting System (BJANC).

On the basis of introducing the core algorithm developed by the Beijing Institute of Urban Meteorology, RMAPS-IN sets up the objective analysis of three-dimensional gridded meteorological elements in the Heilongjiang region with a 1-kilometer 
spatial resolution and 10-minute update cycle in the next $0-12$ hours. Some updated technical improvements including the multi-source observation and multi-system product fusion technology, complex terrain model correction technology and dynamic downscaling technology are integrated in RMAPS-IN system.

VIPS combines both the early warning production and release module which is well integrated with the actual local business. BJANC provides real-time observations of the basic radars but does not have a friendly user interface with relatively simple product and function.

Therefore, it is necessary to establish an intensive, intelligent and integrated platform that integrates all the functions of live monitoring, alarming, nowcasting, short-time forecast and severe weather warning.

\section{Analysis of the Operational Workflow}

The operational workflow of the severe convective weather short-term warning system is mainly based on the prediction of weather and intelligent early warning with 0 to 12 hour lead times through the integration of intelligent platform to make automatic alarm and relevant products, along with the processes of forecast, early warning and verifying to achieve the ability of intelligent alarm and early warning. The main operational process is shown in Figure 1.

The whole process of the business starts from the quality control of multi-source data including surface and airborne observation data, automatic observation data, radar and wind profiler data, satellite data, lightening data, important weather forecast data and numerical weather prediction data, and then with the combination of nowcast system base data, the analysis of severe convective weather is processed. Nowcast system base date is also used to monitor the convective weather to be served as the threshold judgement for alerting information

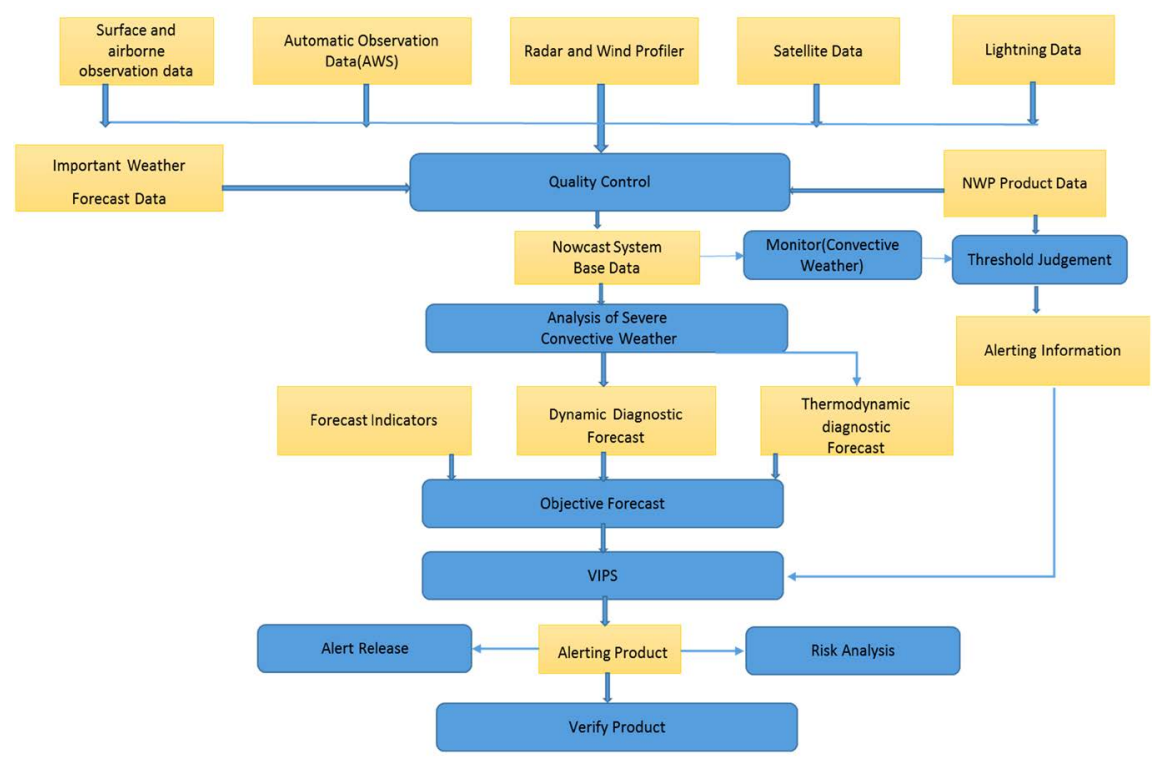

Figure 1. Operational workflow. 
update. With the forecast indicators, dynamic diagnostic forecast and thermodynamic diagnostic forecast, the system makes the objective forecast. By the VIPS system, the alerting product is produced for alert release, risk analysis and product verification.

\section{Interface Design of the System}

The unified design of the project interface is based on the SOA framework. Within the SOA framework, it communicates with the external interface (such as CIMISS, China Integrated Meteorological Information Service System) to form the secondary encapsulation of the external interface. For the local data, the form of reading and writing access to the database and file base is realized through the design interface. For meteorological products, the algorithm execution is carried out in the SOA layer for automatic production, and users can directly access the final product through the interface (Figure 2).

\section{Technical Solutions}

By the establishment of the integrated platform for the three-tier of province-city-county, it not only enhances the real-time display, analysis and warning of the multi-source stereoscopic observation data but also improves the ability of rapid alert products' distribution based on real-time monitoring, implementing the rapid alert production of operational process in a two-tier guidance structure including the province, city and county and seamless link to national emergency warning service platform.

The system effectively improves the accuracy and timeliness of short-time forecast within the Heilongjiang province, and the integration of short-time

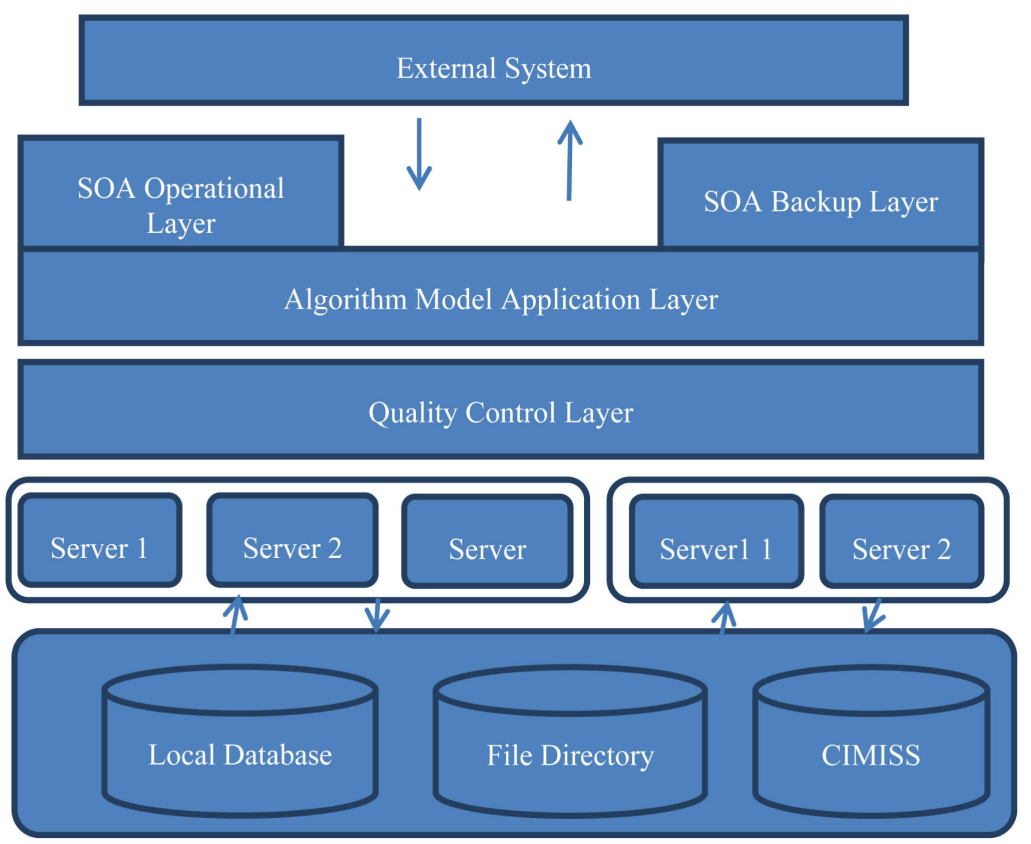

Figure 2. System interface design guidelines. 
forecast business will be achieved at the provincial, municipal and county levels, which enhance the efficiency by realizing intensification, modernization and intellectualization of short-time forecast.

The system fully takes the CMISS data environment into account, linking seamlessly to the existing businesses, with high expansion flexibility to the grid forecast business.

\subsection{Data Support}

\subsubsection{Build a Unified Data Environment and Interface Based on CIMISS Data}

Based on CIMISS data interface, the system establishes a data environment with unified format, naming standards and three tier of province-city-county shared data mechanism for the integrated platform's data support.

Based on CIMISS data environment, the whole workflow of data real-time acquisition, input, call and post-processing is established.

The main functions include as below:

1) To provide data support for short-term forecast early warning system and provincial-municipal-county short-time integration platform;

2) The forecast products and service support products provided by the provincial-municipal-county integration platform can enter the CMISS Data environment;

3) To build a richer data service interface according to the business requirements of the short-time integrated platform of provincial-municipal-county and meet the needs of different business scenarios in the way of Meteorological Unified Service Interface Community (MUSIC) [2] interface;

4) To support the CIMISS access to grid prediction products;

5) To seamlessly link to Heilongjiang integrated platform through data and product exchange.

\subsubsection{Real-Time Data Processing}

On the basis of the unified data environment and interface, according to the actual business requirements of the short-time forecast system and the short-time integrated platform of province-city-county, the real-time automatic data processing on the server side can produce the required format files of radar, automatic station for the short-time forecast system and a large number of picture products combined with WEBGIS display for the short-time integration platform of provinces, cities and counties.

\subsection{Automatic Nowcasting System}

Automatic nowcasting system is based on distributed fast multi-step processing of radar raw data and the techniques of automatic identification, tracking, analysis of thunderstorm weather and 0 - 2 hours automatic nowcasting. The following core functions are achieved through the introduction, localization and adaptation of the system. 
1) Real-time reception, format conversion and quality control algorithm of the new generation weather radar level-II raw data.

2) The CINRAD-SA Doppler radar (also called WSR-98D) [3], SB and CB model radar (made by the Beijing Metstar Radar Co. LTD) has developed a mature program for real-time conversion and decoding of the raw data.

3) Other radar models need to develop programs for converting raw radar data format.

4) Multi-radar intensity echo (including: three-dimensional reflectivity factor and combination reflectivity factor digital jigsaw algorithm).

5) Thunderstorm identification, tracking analysis and nowcasting algorithm.

6) Radar echo cross correlation tracking and extrapolation prediction algorithm.

7) Quantitative precipitation estimation and nowcasting algorithm based on radar.

8) The Chinese version of the Interactive Data Display tool software (C-CIDD) is used to display the real situation, fusion and interpolation analysis results received in real time for various bureau data, intermediate results of each thunderstorm analysis and proximity prediction algorithm, and the final thunderstorm proximity, etc.

\subsection{VIPS}

The goal of VIPS is to enable forecasters to have the latest and most accurate information in the first place to conduct timely analysis and forecasting, making an effort to release weather warning signals to society as soon as possible. The platform is an integrated intelligent platform with a set of live monitoring, automatic alarm, live weather sharing, joint defense of upstream and downstream, superior and subordinate interaction and early warning signal production and release.

This platform uses the technical framework of the combination of CS and BS. The so-called combination refers to the programmer who can use BS structure to develop with the advantages of being more flexible and simple, whereas the program is presented by CS. For the user, it is a CS program without installment.

1) The perfect combination of $C / S$ and $B / S$. The use of the latest technology of NODE + WEBKIT enables the perfect combination of web development and desktop development which gives VIPS the advantage of displaying Web pages and calling client resources, such as reading client files, executing client programs, and so on.

2) Upgraded the efficiency of displaying large capacity of grid data. Using the CANVAS method, the fast probe function of large capacity grid data (such as $1000 \times 1000$ lattice point) is presented in the form of picture.

3) Simplify platform operations. By summing up the use habits of forecasters over the years, the interface function is carefully designed to greatly simplify the operation steps and highlight the weather focus.

The architecture of the platform is as follows (Figure 3). 


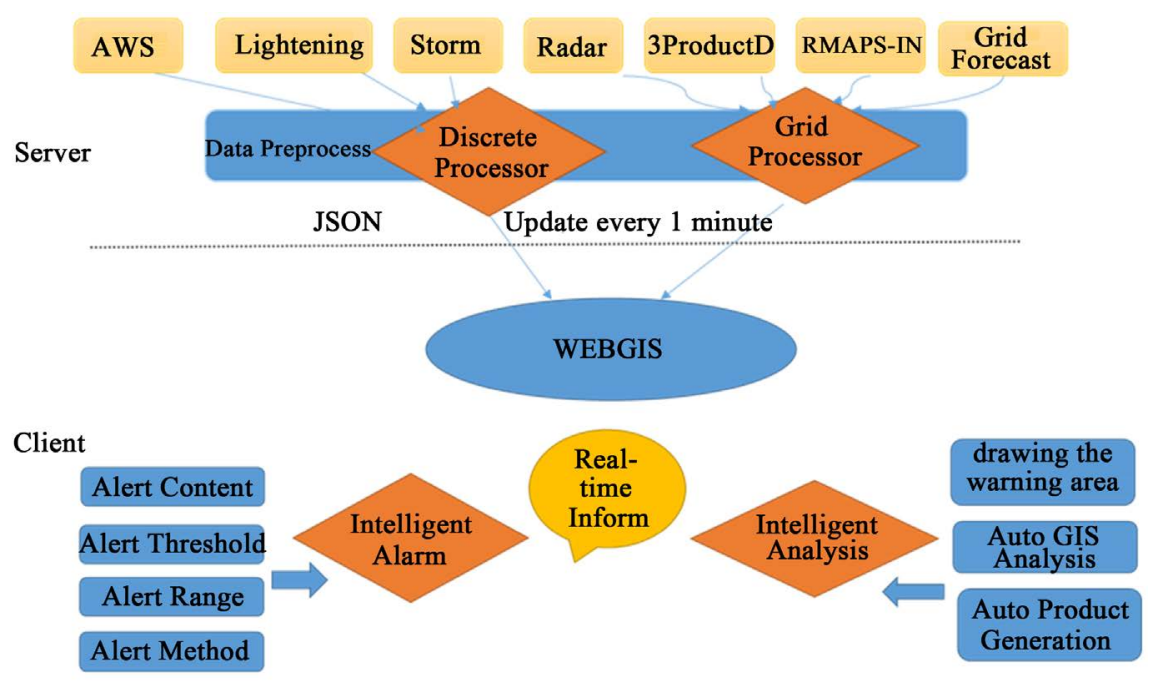

Figure 3. The architecture of the platform.

The functions of the platform are shown as follows:

1) Real-time display and analysis of radar and automatic weather stations (AWS).

2) Automatic reminders of sensitive weather, including radar alarms and AWS warnings, etc.

3) Production and distribution of zoning early warning signals, streamline production processes and operational steps, and support provincial-municipal-county three-tier users to quickly produce and share early warning signals on the same platform.

4) Through the interaction with WEBGIS, the whole process of drawing the warning area, geographic information extraction and final warning product rapid generation and release can be achieved intelligently.

5) Online communication, consultation functions.

6) Historical data query Playback function.

7) Intelligent Automatic alarm and warning remind by phone and SMS.

8) Process monitoring and trace free management.

9) Airborne observation analysis function for live situation and weather forecast.

10) Improve the connection of the early warning products and national emergency warning service platform.

\section{Preliminary Evaluation}

In this section, we present the results of the system. The improvements of RMAPS-IN system mainly focus on the real-time fusion of Heilongjiang area short-time numerical weather prediction system, severe convective weather nowcasting products, RMAPS-IN parameters and program improvement, data interface program, real-time display, product format and business aspects such as 
the integration of the platform to achieve operational RMAPS-IN real-time system, aiming to carry out preliminary testing and evaluation, to provide $1 \mathrm{~km}$ resolution, 10 minutes update frequency of the Heilongjiang region grid ground meteorological data requirements.

1) By using the elevation difference correction method of the near ground elements under the actual complex terrain and the mode terrain, the resolution is improved from $3 \mathrm{KM}$ to $1 \mathrm{KM}$.

2) Improve real-time analysis technology of $1 \mathrm{~km}$ high resolution three-dimensional basic meteorological factor field in Heilongjiang area: based on the minimum demand correction algorithm of temperature and humidity of 2 meters, the grid-based three-dimensional analysis of temperature and humidity mesh with horizontal resolution of $1 \mathrm{~km}$ and vertical resolution of 200 meters in Heilongjiang.

3) Update real-time operation and data environment construction of rapid refresh seamless integrated system in Heilongjiang area: based on the live observation data of 10-minute automatic station of Heilongjiang Meteorological Bureau, this system solves the complex situation of multi-source data, realizes the data format transformation, unifies file format, and provides reliable data service. For complex transmission status, it effectively solves the real-time transmission strategy and format conversion module of the high-density automatic observation station data which must be operated in real time. The existing radar base data is optimized and maintained to ensure its normal, reliable and stable operation to generate products.

4) Establish and improve the real-time operation framework of rapid refresh seamless integrated system in Heilongjiang area: by localizing RMAPS-IN core technology and integrating with Heilongjiang data environment, the system helps to establish high resolution grid seamless rapid integrated weather forecasting system in Heilongjiang.

\section{Conclusion}

In summary, the system is featured with the products with high resolution (1 $\mathrm{km}$ ), high timelessness (updated by 10 minutes), which can provide refined analysis of the ground and upper air as well as the refined forecast in the next 12 hours, serving as the reference products for automatic nowcasting. On the other hand, during the construction of the system involving data and application requirements from different departments, not all the staff members engage in it, which leads to the constraints of resources and technology. It could be better if all members from various related departments would participate in the project.

\section{Acknowledgements}

The project presented in this paper is supported by the construction project of MABIS (Management and Business Integration System). 


\section{Conflicts of Interest}

The authors declare no conflicts of interest regarding the publication of this paper.

\section{References}

[1] Su, D.B., Xu, W.J., Li, Q.C. and Shen, Y.H. (2012) Application and Development Trend of Short-Time Proximity Forecast Pre-System (VIPS, Very-Short-Range Interactive Prediction System). China Science and Technology Achievements, No. 13, 32-34.

[2] Ji, Y.H., Sun, C., Liu, Y.M. and Xiao, W.Q. (2017) A Method of Optimizing Storage Efficiency of Meteorological Data in CIMISS. Meteorological Science and Technology, 45, 30-35.

[3] Sheng, C.Y., Gao, S.T. and Xue, M. (2006) Short-Range Prediction of a Heavy Precipitation Event by Assimilating Chinese CINRAR-SA Radar Reflectivity Data Using Complex Cloud Analysis. Meteorology and Atmospheric Physics, 94, 167-183. https://doi.org/10.1007/s00703-005-0177-0 